УДК 541.13:541.183.12

\title{
ВЛИЯНИЕ ДИСПЕРСНОСТИ СУЛЬФОКАТИОНООБМЕННИКА ГЕТЕРОГЕННЫХ МЕМБРАН НА РАЗВИТИЕ ДИФФУЗИОННЫХ ПОГРАНИЧНЫХ СЛОЕВ ПРИ ИНТЕНСИВНЫХ ТОКОВЫХ РЕЖИМАХ
}

\author{
() 2018 Э. М. Акберова, Д. В. Костылев, В. И. Васильева \\ Воронежский государственный университет, Университетская пл., 1, 394018 Воронеж, Россия \\ e-mail: elmara_09@inbox.ru
}

Поступила в редакцию 27.07.2018

\begin{abstract}
Аннотация. Электрическая неоднородность поверхности гетерогенных инообменных мембран Ralex CM Pes (MEGA a.s., Чехия) при варьировании времени измельчения ионообменника определяет интенсивность электроконвективного перемешивания раствора на межфазной границе и параметры диффузионных слоев. С ростом времени измельчения частиц ионообменника установлено увеличение степени дисперсности, уменьшение величины шага электрической неоднородности (суммарного размера проводящей и непроводящей фаз) поверхности, доли макропор и сглаживание микрорельефа гетерогенных сульфокатинообменных мембран. С ростом времени измельчения ионообменника от 5 до 80 мин установлено снижение скачка потенциала мембраны в полтора раза, при котором начинается уменьшение эффективной толщины диффузионного слоя. Выявлена общая тенденция снижения общей и эффективной толщин диффузионного слоя с уменьшением шага электрической неоднородности поверхности мембран.
\end{abstract}

Ключевые слова: гетерогенная сульфокатионообменная мембрана, неоднородность поверхности, диффузионный пограничный слой, электроконвективная нестабильность, сверхпредельные токовые режимы.

DOI: https://doi.org/10.17308/kcmf.2018.20/572

\section{ВВЕДЕНИЕ}

Развитие концепции диффузионного слоя (ДС) и определение его параметров представляет большой интерес для понимания процессов переноса в электромембранных системах. Размеры диффузионного пограничного слоя (ДПС) являются одной из важнейших кинетических характеристик диффузионной кинетики, поскольку определяют величину плотности потока вещества на межфазной границе:

$$
\delta_{N}=\frac{D\left(C_{0}-C_{s}\right)}{J},
$$

где $D$ - коэффициент диффузии электролита, $C_{s}-$ концентрация вещества на границе раствор-мембрана, $C_{0}$ - концентрация вещества за пределами диффузионного слоя, $\delta_{N}-$ эффективная толщина диффузионного слоя, $J$ - поток вещества. Уменьшение толщины диффузионного слоя приводит к повышению скорости процесса.
Теоретически оценить эффективную толщину ДПС позволяет уравнение Пирса [1] для предельной плотности тока $i_{\lim }$ :

$$
i_{\lim }=\frac{F D C_{0}}{\delta\left(T_{1}-t_{1}\right)} .
$$

В уравнении (2) $D_{0}$ - коэффициент диффузии электролита, $C_{0}-$ концентрация электролита на входе в канал обессоливания, $F$ - число Фарадея, $\delta$ - толщина ДПС, $T_{1}$ и $t_{1}$ - число переноса противоионов в мембране и растворе, соответственно. Для относительно коротких каналов, состоящих из двух параллельных пластин, толщина диффузионного слоя Нернста в растворе может быть рассчитана также по уравнению Левека в соответствии с теорией конвективной диффузии [2]:

$$
\delta_{\text {Lev }}=0.71 \mathrm{~h}\left(\frac{L D}{h^{2} V_{0}}\right)^{1 / 3},
$$


где $L$ - длина пути обессоливания, $h$ - межмембранное расстояние, $V_{0}$ - линейная скорость протока раствора.

Экспериментально структура диффузионного слоя может быть исследована с помощью различных косвенных методов: путем обработки вольтамперных кривых [3, 4], хронопотенциограмм $[4,5]$ и спектров электрохимического импеданса $[6,7]$. Прямым экспериментальным методом определения толщины ДС является лазерная интерферометрия [8-11]. Результаты теоретических расчетов с использованием двумерной “базовой” 2Dмодели переноса ионов и воды в электродиализной ячейке с учетом электроконвекции, предложенной М. Х. Уртеновым и др. [12], достоверно согласуются с экспериментальными значениями толщин диффузионных слоев, найденными из интерферограмм [13].

Толщина ДС в мембранных системах зависит от многих факторов. Наиболее существенными являются гидродинамика течения [11,14-17] и геометрия канала $[8,9,15,17-19]$. К очевидным способам уменьшения толщины диффузионного пограничного слоя относятся увеличение скорости подачи растворов, ускоряющее доставку ионов к поверхности, и использование спейсеров в межмембранном пространстве, облегчающее перемешивание раствора.

В работе [17] установлено, что рост линейной скорости подачи растворов хлорида натрия разной концентрации вызывает снижение толщины диффузионного слоя более чем в 2.5 раза. Шестикратное увеличение скорости протока раствора приводит к уменьшению толщины ДПС в растворе на границе с катионо- и анионообменными мембранами в 1.7 и 1.4 раза соответственно [15]. Введение в канал спейсеров более эффективно: толщины ДПС уменьшаются в 1.7-2.3 раза по сравнению с гладкими каналами [17]. Методом лазерной интерферометрии установлено прерывание диффузионного слоя в канале с ионообменными спейсерами: увеличение толщины диффузионных пограничных слоев перед спейсером, а затем резкое уменьшение $[8,9,18]$. Использование спейсеров из инертного материала приводит к увеличению падения напряжения и сокращению рабочей поверхности [20], что в каналах как с катионообменными, так и анионообменными мембранами сопровождается ростом толщины диффузионного слоя в растворе на 20-60 и 30-80 \% соответственно [15]. Однако замена авторами [15] инертных турбулизирующих вставок на ионопроводящие не позволило сущест- венно интенсифицировать процесс: для катионообменных мембран использование данных вставок в канале сопровождается уменьшением толщин ДПС на 2-18\%, в то время как в случае анионообменных мембран размеры диффузионного слоя увеличиваются на $1-27 \%$.

В системах с ионообменными мембранами толщина ДС может зависеть от свойств поверхности. В работе [4] для гомогенной мембраны Nafion ycтановлено уменьшение определенной по данным вольтамперометрии толщины диффузионного слоя в 1.5 раза по сравнению с гетерогенной мембраной МК-40. Данный факт авторами был объяснен возможной зависимостью толщины ДС от электрической неоднородности и гидрофобности поверхности мембраны. Согласно расчетам, проведенным в [19], наличие на гладких мембранах прямоугольных и треугольных по форме ионопроводящих выступов высотой от 25 до 300 мкм способствовало уменьшению толщины диффузионного слоя. В работе [21] показано, что изменение свойств поверхности мембран (электрическая и геометрическая неоднородность) в ходе температурного модифицирования определяет параметры диффузионных слоев при плотностях тока, превышающих предельные диффузионные. Известно небольшое количество работ, посвященных закономерностям формирования диффузионных слоев в зависимости от степени неоднородности поверхности ионообменных мембран, что определило задачи настоящей работы. Целью работы является лазерно-интерферометрическое изучение влияния разной степени дисперсности сульфокатионообменника экспериментальных образцов гетерогенных мембран Ralex CM Pes на толщины диффузионных слоев в растворе на межфазной границе.

\section{ЭКСПЕРИМЕНТАЛЬНАЯ ЧАСТЬ}

Объектами исследования были выбраны экспериментальные образцы гетерогенных сульфокатионообменных мембран Ralex CM Pes («MEGA» a.s., Чехия), которые были получены вальцеванием гомогенизированной смеси измельченного ионообменника различной степени дисперсности с полиэтиленом. Объемное соотношение катионита и полиэтилена сохранялось при этом одинаковым. Объемная доля ионообменной смолы в смеси, используемой при изготовлении серии мембран CM Pes, составляла 58 \%. Степень дисперсности сульфокатионообменника варьировалась путем использования разного времени его измельчения от 5 до 80 мин. Образцы исследуемых 
мембран подвергали стандартной солевой предподготовке [22].

Эксперименты по изучению формирования и развития концентрационных полей на межфазной границе при интенсивных токовых режимах были выполнены в семисекционной электродиализной ячейке. Исследуемый мембранный канал состоял из однотипных сульфокатионообменных мембран. Высота мембранного канала $L$ составляла $4.1 \cdot 10^{-2} \mathrm{M}$, ширина $1.4 \cdot 10^{-2} \mathrm{M}$, межмембранное расстояние $h=2.0 \cdot 10^{-3}$ м. Раствор 0.02 М хлорида натрия в исследуемую секцию подавался со скоростью $1.3 \cdot 10^{-3} \mathrm{M}(R \mathrm{e}=2.6)$. Электродиализ был проведен в гальваностатическом режиме при горизонтальной ориентации электродиализатора в гравитационном поле (устойчивая концентрационно-температурная стратификация).

Для исследования диффузионных слоев в электромембранных системах использовали установку

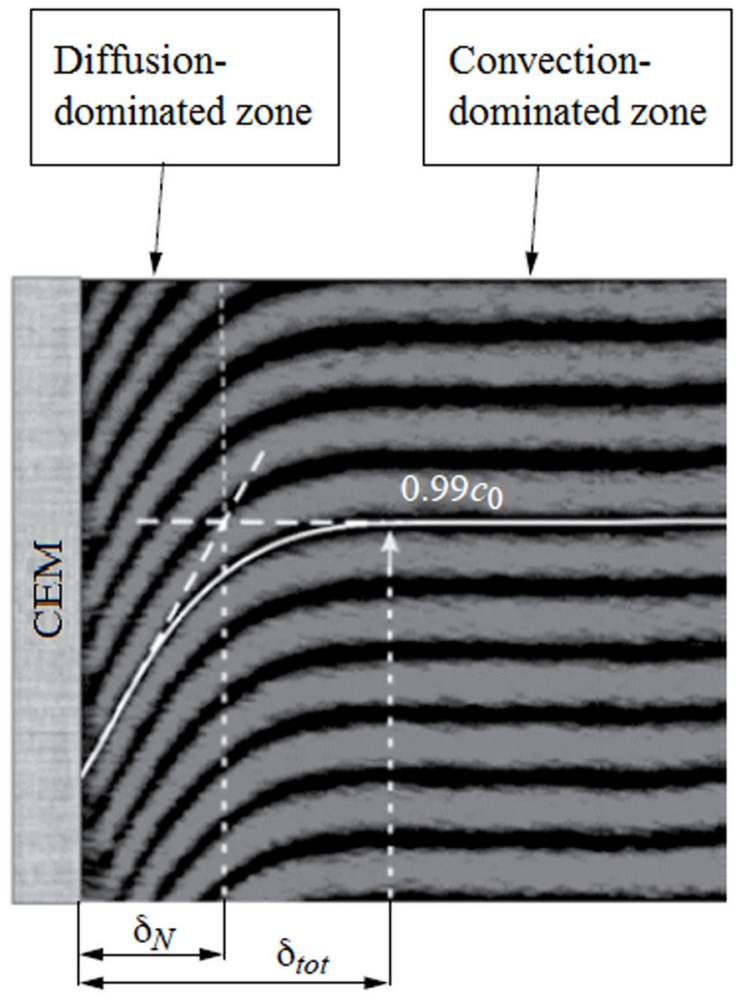

$a$ по схеме Маха-Цендера. Схема, методы получения и декодирования интерферограмм описаны в [11, $16,23]$. Интерферограммы снимались на координате по направлению подачи раствора $y=0.64 L$.

В качестве общей толщины диффузионного слоя $\delta_{\text {tot }}$ принималось расстояние от границы раздела до точки в растворе с концентрацией равной 0.99 от концентрации за его пределами (рис. 1a). Толщина диффузионного слоя Нернста $\delta_{\mathrm{N}}$ определялась как расстояние от межфазной границы до точки пересечения касательных к концентрационному профилю на границе раздела фаз и в глубине раствора. При сверхпредельных токовых режимах толщина диффузионного слоя Нернста $\delta_{\mathrm{N}}$, согласно $[13,24]$, была получена пересечением касательных к профилю концентрации, проведенных на границе области электроконвективной нестабильности $d$ и зоны устойчивого концентрационного распределения (рис. $1 b)$. Размер области конвективной неста-

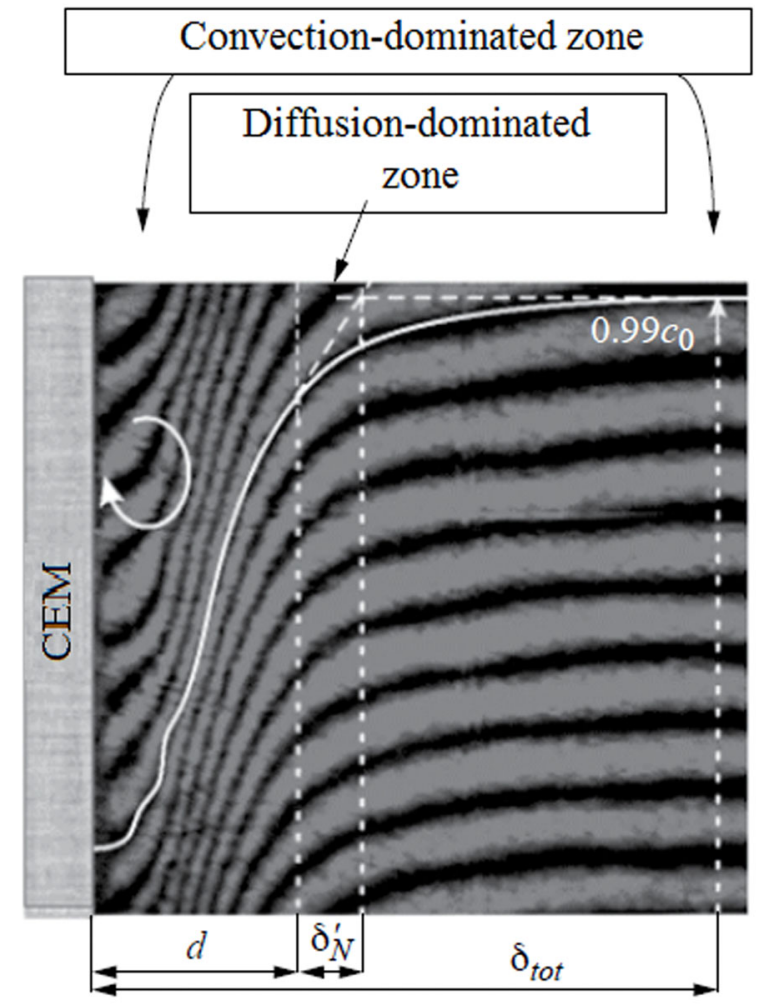

$b$

Рис. 1. Интерферограммы, отображающие концентрационные профили в растворе на границе с катионообменной мембраной при допредельной $i=0.5 i_{\lim }(a)$ и сверхпредельной $i=2 i_{\lim }(b)$ плотностях тока. $\delta_{\mathrm{tot}}-$ полная толщина диффузионного слоя; $\delta_{N}-$ эффективная локальная толщина диффузионного слоя Нернста при $i<i_{\lim }$; толщины области с доминирующим диффузионным механизмом доставки ионов $\delta{ }_{N}{ }_{N}$ и области электроконвективной нестабильности $d$ при $i>i_{\text {lim }}$. Адаптировано из [27]

[Fig. 1. Interferometric bands visualizing concentration profiles in solution on the boundary with cation-exchange membrane at underlimiting $i=0.5 i_{\lim }(a)$, and overlimiting $\mathrm{i}=2 i_{\lim }(b)$ current densities. $\delta_{\text {tot }}$ is the total diffusion layer thickness; $\delta_{N} \mathrm{t}$ is the effective Nernst's local diffusion layer thickness at $i<i_{\text {limm }}$; the thicknesses of the diffusion-dominated zone $\delta{ }_{N}$ and of the electroconvective instability region $d$ at $i>i_{\text {lim }}$. Adapted from [27]] 
бильности $d$ определялся как расстояние от поверхности мембраны до точки в растворе, на котором интерференционная полоса и, соответственно, концентрационный профиль имели нестационарный, колебательный характер [21, 25, 26].

При измерении вольтамперной характеристики исследуемой мембраны использовались два хлоридсеребряных зонда, расположенные по обе стороны на расстоянии 1.3 мм от ее поверхности. При построении ВАХ вместо суммарного скачка потенциала $\Delta \varphi$ использовали величину приведенного скачка потенциала $\Delta \varphi^{\prime}[28,29]$ :

$$
\Delta \varphi^{\prime}=\Delta \varphi-i R_{e f}
$$

где $\Delta \varphi$ - измеряемая величина суммарного скачка потенциала при плотности тока $i, R_{e f}\left(\mathrm{Oм}^{\circ} \mathrm{cm}^{2}\right)$ - эффективное сопротивление мембранной системы, определяемое по наклону начального участка вольтамперной кривой при низких значениях плотности электрического тока $(\partial \Delta \phi / \partial i)_{i \rightarrow 0}$.

Исследования морфологии поверхности набухших мембран проводили методом растровой электронной микроскопии с применением микроскопа марки JSM-6380 LV (Япония) с регулируемым давлением в камере с исследуемым образцом [30, 31]. Количественная оценка доли и размеров ионообменного материала и макропор на поверхности мембран проводилась с помощью авторского программного комплекса [32].

\section{РЕЗУЛЬТАТЫ И ИХ ОБСУЖДЕНИЕ}

Структура поверхности исследуемых гетерогенных катионообменных мембран Ralex является неоднородной: визуализируются участки проводящей электрический ток поверхности (частицы ионообменника) и инертные участки полиэтилена, соответствующие непроводящей поверхности. Установлено, что с увеличением продолжительности измельчения ионита соотношение проводящей и непроводящей фазы на поверхности мембран сохраняется постоянным. Доля ионообменника на поверхности экспериментальных мембран Ralex в набухшем состоянии составляет 15-17\%. C ростом времени измельчения ионообменника от 5 до 80 мин выявлено уменьшение размеров проводящих участков на $20 \%$, увеличение их общего количества и значительное возрастание доли ионообменных частиц с размером менее 1.4 мкм на поверхности мембран (рис. 2a). Согласно проведенным в работе [33] оценкам для выпускаемых в промышленном масштабе компанией «MEGA» a.s. (Чехия) мембран Ralex CM Pes нами установлено, что размеры участков выхода ионообменника на поверхность мембраны - от 1 до 14 мкм, значение их средневзвешенного радиуса равно $\bar{R}=1.88$ мкм.

Увеличение продолжительности измельчения ионообменных частиц соответствует росту их степени дисперсности: для образцов мембран с измельчением ионообменника в течение 5 и 80 мин разброс величин степени дисперсности составляет (3-80) $\cdot 10^{4} \mathrm{M}^{-1}$ и (7-125) $10^{4} \mathrm{M}^{-1}$ соответственно. Установлено, что с ростом степени дисперсности ионообменных частиц средневзвешенная величина расстояния между ними уменьшается на 40 \%. При этом на поверхности мембран при времени измельчения ионообменника 80 мин минимальная протяженность непроводящих участков составляет менее 1 мкм, а большая часть ионообменных частиц находится на расстоянии от 1 до 8 мкм (рис. $2 b$ ). Шаг электрической неоднородности, являющийся характеристикой неоднородности поверхности мембран и равный сумме средневзвешенных величин проводящего и непроводящего участков, линейно уменьшается от 13.0 до 8.5 мкм с увеличением времени измельчения ионообменника от 5 до 80 мин.

Сравнение АСМ-изображений поверхности экспериментальных мембран показало, что мембрана с большей степенью дисперсности ионообменника, соответствующей времени измельчения 80 мин, характеризуется гладкой в микрометрическом масштабе поверхностью. Для мембран со временем измельчения ионообменника 5 и 80 мин размах высот составлял 486 и 286 нм соответственно. Установлено, что размах высот микропрофиля поверхности мембраны с меньшей степенью дисперсности ионита гораздо больше не только за счет размеров частиц ионообменника, но и более развитой пористости. Уменьшение продолжительности измельчения частиц ионита приводит к увеличению поверхностной пористости на $26 \%$.

В соответствии с теорией конвективной диффузии по уравнению (3) была рассчитана толщина диффузионного слоя $\delta_{\mathrm{Lev}}$ в растворе на границе с катионообменной мембраной. Данная величина составила 331 мкм. Установлено, что в области допредельных и предельных токов для исследуемых экспериментальных образцов мембран Ralex CM Pes c разным временем измельчения ионообменника в растворе хлорида натрия толщина ДС меньше своего теоретического значения.

На рис. 3 представлены экспериментально полученные зависимости общей $\left(\delta_{\text {tot }}\right)$ и эффективной $\left(\delta_{\mathrm{N}}\right)$ толщин диффузионного слоя, а также размера 

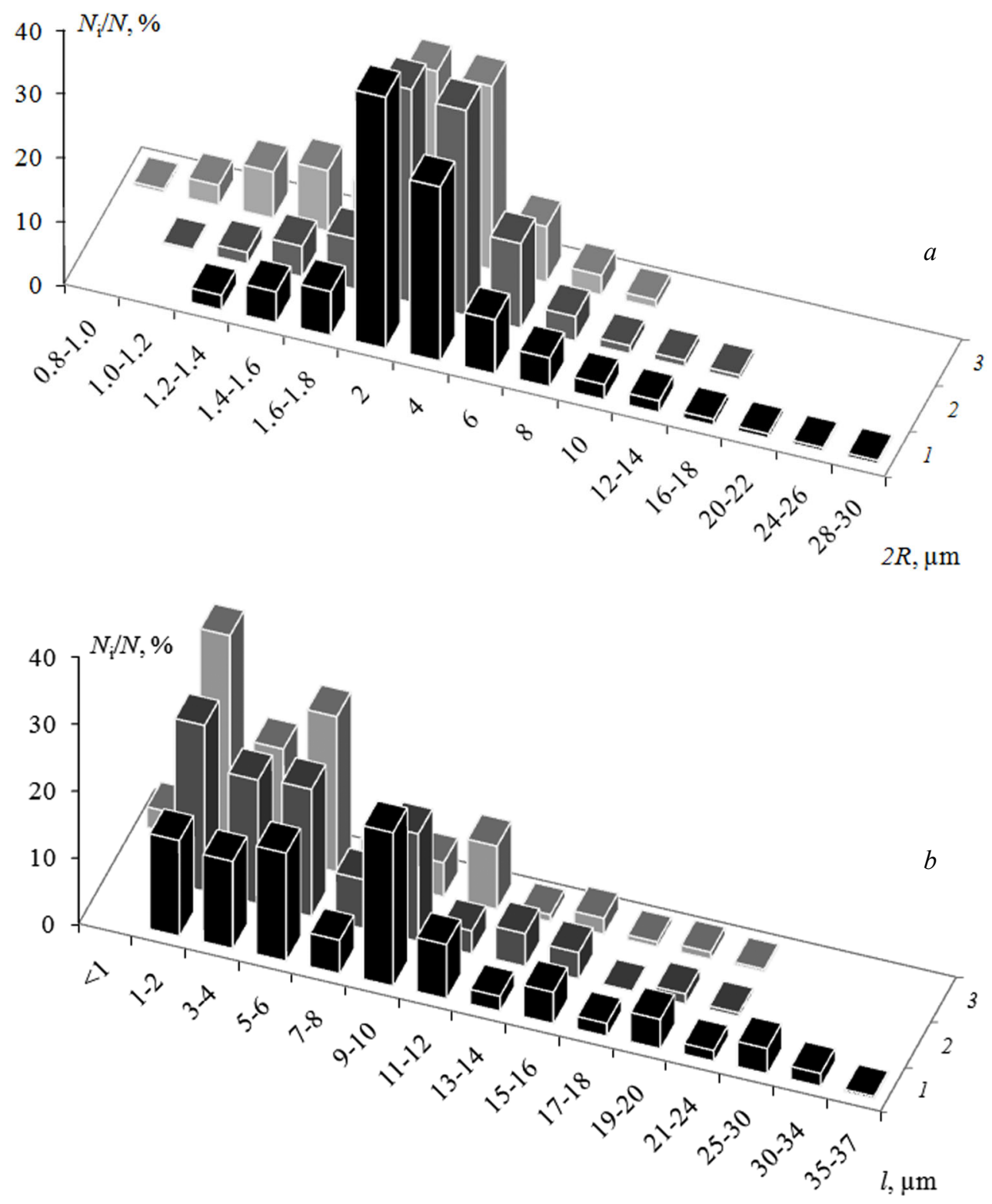

Рис. 2. Распределение по размерам участков ионообменника $(a)$ и расстояния между ними $(b)$ на поверхности набухших образцов ионообменных мембран Ralex CM Pes при времени измельчения ионита 5 (1), 40 (2) и 80 (3) мин

[Fig. 2. Distribution of the ion-exchange regions along the sizes $(a)$ and the distances between them $(b)$ on the surface of the swollen samples of ion-exchange membranes Ralex CM Pes for the milling time of the ion-exchanger 5 (1), 40 (2) and $80(3) \mathrm{min}]$

области электроконвективной нестабильности $(d)$ от приведенного скачка потенциала. С увеличением плотности тока экспериментально обнаружены рост области конвективной нестабильности раствора у поверхности мембраны и общей толщины $\delta_{\text {tot }}$, но уменьшение толщины диффузионного слоя Нернста $\delta_{\mathrm{N}}$. Когда размеры области конвективной нестабильности соответствуют общей толщине пограничного слоя, происходит полное разрушение диффузионного слоя.
С ростом времени измельчения ионообменника от 5 до 80 мин установлено снижение скачка потенциала мембраны, при котором начинается уменьшение эффективной толщины диффузионного слоя. Полное разрушение диффузионного слоя в растворе на границе с мембраной при времени измельчения ионообменных частиц 80 мин зафиксировано при приведенном скачке потенциала в 1.7 раза меньше, чем в случае с мембраной при продолжительности измельчения ионита 5 мин (рис. 3). Дан- 


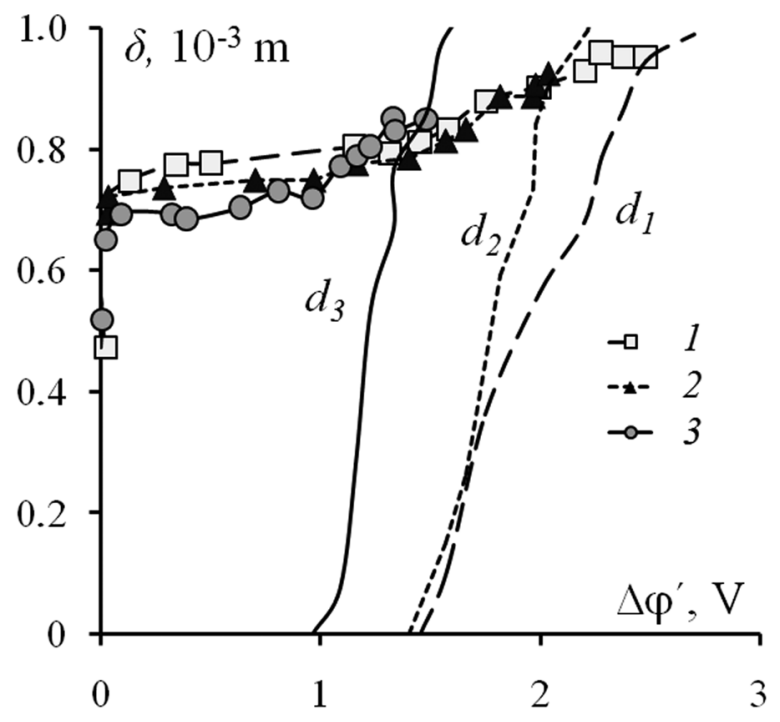

$a$

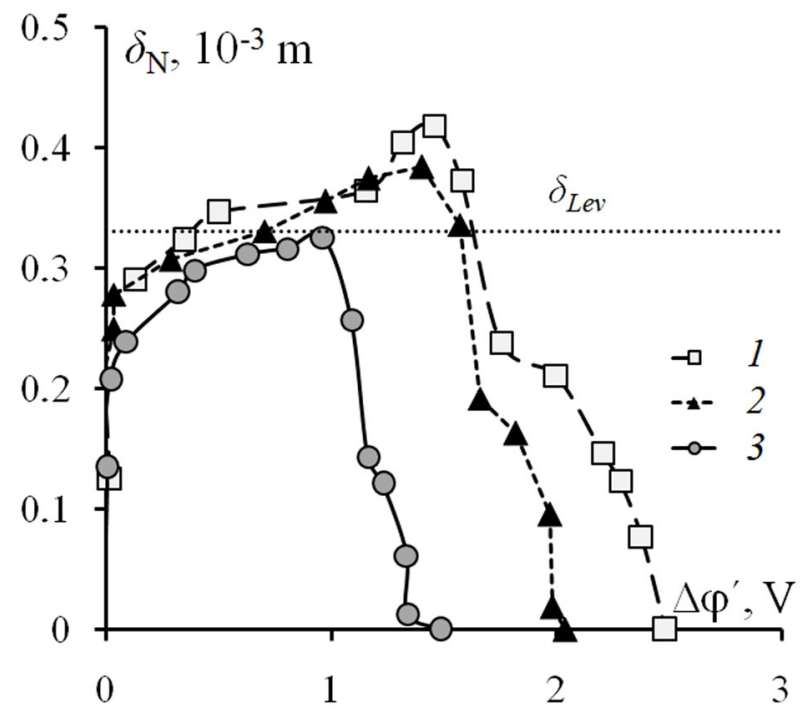

$b$

Рис. 3. Зависимость общей $(a)$, эффективной $(b)$ толщин диффузионного слоя и размера области конвективной нестабильности $(d)$ в растворе на границе с экспериментальными сульфокатионообменными мембранами Ralex $\mathrm{CM}$ Pes от приведенного скачка потенциала $\Delta \varphi^{\prime}: C_{0}(\mathrm{NaCl})=2.0 \cdot 10^{-2} \mathrm{M}, V=1.3 \cdot 10^{-3} \mathrm{M} / \mathrm{c}, h=2.0 \cdot 10^{-3} \mathrm{M}, y=2.6 \cdot 10^{-2} \mathrm{M}$

(0.64L). Время измельчения ионообменника: 5 (1), 40 (2) и 80 (3) мин

[Fig. 3. The dependence of the total $(a)$, effective $(b)$ thicknesses of the diffusion layer and the size of the convective instability region $(d)$ in the solution at the boundary with the experimental sulfocation-exchange membranes Ralex CM Pes from reduced potential drop: $C_{0}(\mathrm{NaCl})=2.0 \cdot 10^{-2} \mathrm{M}, V=1.3 \cdot 10^{-3} \mathrm{~m} / \mathrm{s}, h=2.0 \cdot 10^{-3} \mathrm{~m}, y=2.6 \cdot 10^{-2} \mathrm{~m}(0.64 \mathrm{~L})$. Ion-exchanger milling time: $5(1), 40(2)$ and $80(3) \mathrm{min}]$

ный факт обусловлен более интенсивным электроконвективным перемешиванием раствора на границе с мембраной Ralex CM Pes с максимальной степенью дисперсности частиц ионообменника, хотя и минимальной шероховатостью поверхности среди исследуемых образцов мембран.

Выявлена корреляция между параметрами диффузионных слоев и шагом электрической неоднородности поверхности мембран (рис. 4). При увеличении времени измельчения и, соответственно, уменьшении шага электрической неоднородности поверхности установлено снижение общей и эффективной толщин диффузионного слоя.

\section{ЗАКЛЮЧЕНИЕ}

Микроскопическими методами выявлены различия свойств поверхности гетерогенных сульфокатионообменных мембран Ralex CM Pes paзной технологии изготовления. С ростом времени измельчения частиц ионообменника от 5 до 80 мин. происходит уменьшение величины шага электрической неоднородности поверхности, доли макропор и сглаживание микрорельефа. Наиболее однородным распределением характеристик поверхнос- ти обладает мембрана, полученная при времени измельчения ионообменника 80 мин.

Методом лазерной интерферометрии измерены толщины диффузионных слоев и области электроконвективной нестабильности в растворе хлорида натрия на границе с экспериментальными сульфокатионообменными мембранами Ralex CM Pes с разной степенью дисперсности ионообменника. Установлено, что изменение микроструктуры поверхности исследуемых мембран при изменении условий технологии их изготовления определяет интенсивность электроконвективного перемешивания на границе мембрана-раствор и параметры диффузионных слоев. С ростом времени измельчения ионообменника, что соответствует уменьшению суммарного размера проводящей и непроводящей фаз на поверхности мембран, установлена тенденция снижения общей и эффективной толщин диффузионного слоя.

Работа выполнена при поддержке гранта Президента Российской Федерации МК-925.2018.3.

Авторы выражают благодарность компании «Mеga» a.s. (Чешская Республика) и г-ну Л. Новаку за предоставленные экспериментальные мембраны Ralex CM Pes. 

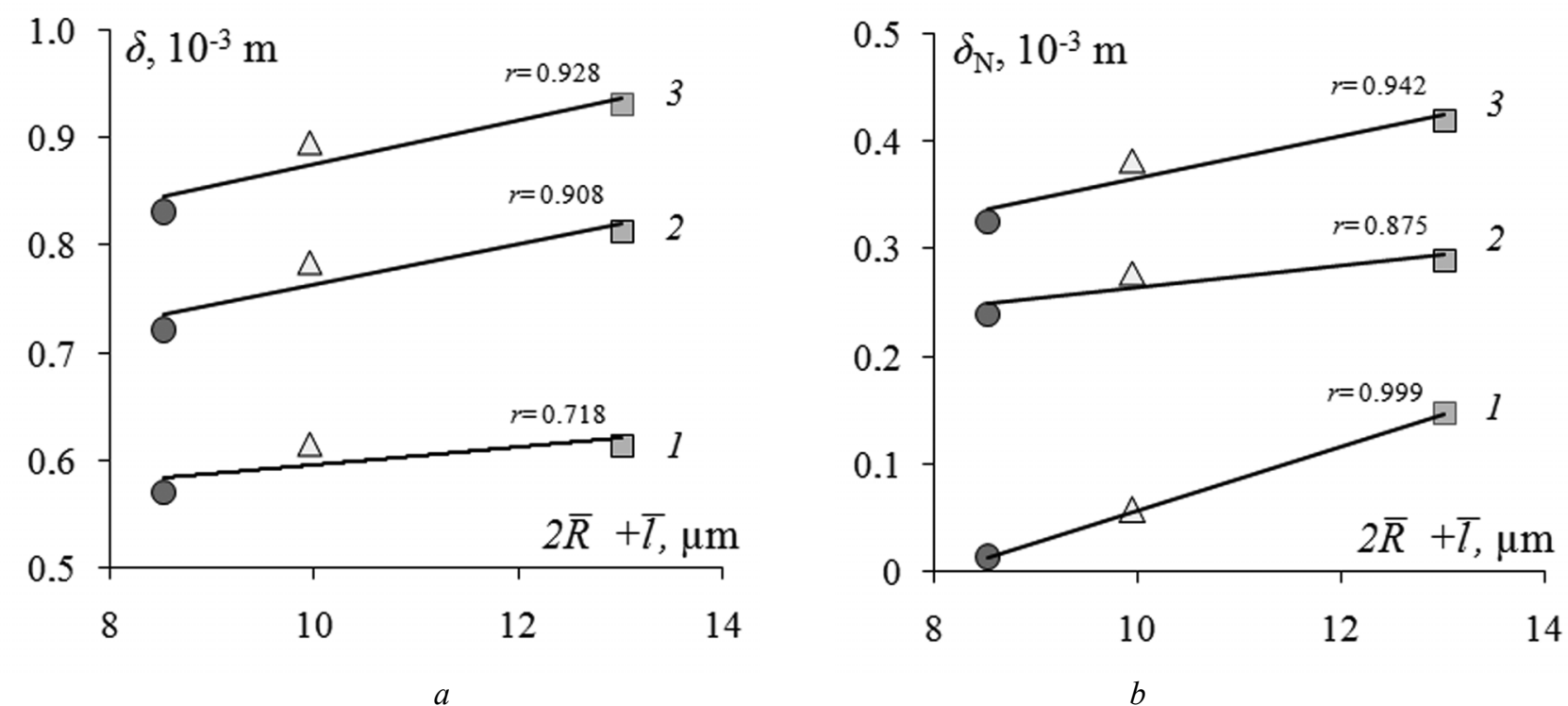

Рис. 4. Зависимость общей $(a)$ и эффективной $(b)$ толщин диффузионного слоя от шага электрической неоднородности поверхности мембран Ralex CM Pes при кратности превышения предельной диффузионной плотности тока: 0.5 (1); 2.0 (2) и 3.0 (3). $r$ - коэффициент корреляции

[Fig. 4. The dependence of the total $(a)$ and effective $(b)$ thicknesses of the diffusion layer from electrical heterogeneity spacing of CM Pes membrane surface at currents that are higher than the diffusion limiting current density by 0.5 (1); 2.0 (2); and 3.0 times (3). $r$ is the correlation coefficient]

Микрофотографии и АСМ-изображения поверхности мембран получены на оборудовании Центра коллективного пользования Воронежскогогосударственного университета. URL: http://ckp.vsu.ru.

\section{СПИСОК ЛИТЕРАТУРЫ}

1. Peers A. M. // Discuss. Faraday Soc., 1956, vol. 21, pp. 124-125. DOI: 10.1039/DF9562100117

2. Newman J., Thomas-Alyea K. E. // Electrochemical Systems. New York, John \& Sons, 2004, 672 p.

3. Urtenov M. A.-Kh., Kirillova E. V., Seidova N. M., Nikonenko V. V. // J. Phys. Chem. B, 2007, vol. 111, № 51, pp. 14208-14222. DOI: $10.1021 /$ jp073103d

4. Козмай А. Э., Никоненко В. В., Письменская Н. Д., Пряхина О. Д., Систа Ф., Пурселли Ж. // Электрохимия, 2010 , т. 46, № 12, с. 1477-1483. DOI: 10.1134/ S1023193510120074

5. Larchet C., Nouri S., Auclair B., Dammak L., Nikonenko V. // Adv. Colloid Interface Sci., 2008, vol. 139, pp. 45-61. DOI: 10.1016/j.cis.2008.01.007

6. Sistat P., Kozmai A., Pismenskaya N., Larchet C., Pourcelly G., Nikonenko V. // Electrochim. Acta, 2008, vol. 53, № 22, pp. 6380-6390. DOI: 10.1016/j.electacta.2008.04.041

7. Мареев С. А., Козмай А. Э., Письменская Н. Д., Никоненко В. В. // Конденсированные среды и межфазные границы, 2011, т. 13, № 2, с. 172-177. http://www. kcmf.vsu.ru/resources/t_13_2_2011_009.pdf

8. Васильева В. И., Шапошник В. А., Григорчук О. В. // Электрохимия, 2001, т. 37, № 11, с. 13391347. DOI: $10.1023 / \mathrm{A}: 1012763531803$
9. Grigorchuk O. V., Vasil'eva V. I., Shaposhnik V. A. // Desalination, 2005, vol. 184, pp. 431-438. DOI: 10.1016/ j.desal.2005.03.048

10. Шапошник В. А., Васильева В. И., Григорчук О. В. // Электрохимия, 2006, т. 42, № 11, с. 13401345. DOI: $10.1134 / \mathrm{S} 1023193506110061$

11. Васильева В. И. // Вестник ВГУ, серия: Химия. Биология. Фармащия, 2007, № 1, с. 359-379.

12. Urtenov M. K., Uzdenova A. M., Kovalenko A. V., Nikonenko V. V., Pismenskaya N. D., Vasil'eva V. I., Sistat P., Pourcelly G. // J. Membr. Sci., 2013, vol. 447, pp. 190202. DOI: 10.1016/j.memsci.2013.07.033

13. Nikonenko V. V., Vasil'eva V. I., Akberova E. M., Uzdenova A. M., Urtenov M. K., Kovalenko A. V., Pismenskaya N. P., Mareev S. A., Pourcelly G. // Adv. Colloid Interface Sci., 2016, vol. 235, pp. 233-246. DOI: 10.1016/j.cis.2016.06.014

14. Pérez-Herranz V., Guiñón J. L., García-Antón J. // J. Applied Electrochem., 2000, vol. 30, iss. 7, pp. 809-816. DOI: $10.1023 / \mathrm{A}: 100402242$

15. Shahi V. K., Thampy S. K., Rangarajan R. // Desalination, 2001, vol. 133 , pp. 245-258. DOI: $10.1016 /$ S0011-9164(01)00105-9

16. Васильева В. И., Шапошник В. А., Григорчук О. В., Малыхин М. Д. // Электрохимия, 2002, т. 38, № 8, c. 949-955. DOI: 10.1023/A:1016809727673

17. Pawlowski S., Sistat Ph., Crespo J. G., Velizarov S. // J. Membr. Sci., 2014, vol. 471, pp. 72-83. DOI: 10.1016/ j.memsci.2014.07.075

18. Shaposhnik V. A., Vasil'eva V. I., Grigorchuk O. V. // Adv. Colloid Interface Sci., 2008, vol. 139, pp. 74-82. DOI: 10.1016/j.cis.2008.01.008 
19. Tadimeti J. G. D., Kurian V., Chandra A., Chattopadhyay S. // J. Membr. Sci., 2016, vol. 499, pp. 418428. DOI: 10.1016/j.memsci.2015.11.001

20. Гнусин Н. П., Заболоцкий В. И., Письменский В. Ф., Литвинов С. П. // Журн. прикл. химии, 1979 , т. 52 , с. $1053-1058$.

21. Акберова Э. М. // Конденсированные среды $и$ межфазные гранищь, 2017, т. 19, № 3, с. 314-320. http:// www.kcmf.vsu.ru/resources/t 1932017 001.pdf

22. Березина Н. П., Кононенко Н. А., Дворкина Г. А, Шельдешов Н. В. Физико-химические свойства ионообменных материалов, Краснодар: Изд-во Кубан. гос. ун-та, 1999. 82 с.

23. Vasil'eva V. I., Shaposhnik V. A., Grigorchuk O. V., Petrunya I. P. // Desalination, 2006, vol. 192, № 1-3, pp. 408-414. DOI: 10.1016/j.desal.2005.06.055

24. Vasil'eva V., Zhiltsova A., Shaposhnik V., Zabolotsky V., Lebedev K., Malykhin M. // "Ion Transport in Organic and Inorganic Membranes". Proceedings of Intern. Conf., 28 May - 2 June, 2012, Krasnodar, 2012, pp. 233235.

25. Васильева В. И., Акберова Э. М., Заболоцкий В. И. // Электрохимия, 2017, т. 53, № 4, с. 452-465. DOI: $10.1134 / \mathrm{S} 1023193517040127$

26. Акберова Э. М., Васильева В. И., Малыхин М. Д. // Конденсированные среды и межфазные гранийы, 2015, т. 17, № 3, с. 273-280. http://www.kcmf. vsu.ru/resources/t_17_3_2015_002.pdf
27. Никоненко В. В., Мареев С. А., Письменская Н. Д., Узденова А. М., Коваленко А. В., Уртенов М. Х., Пурсели Ж. // Электрохимия, 2017, т. 53, № 10, c. 1266-1289. DOI: 10.1134/S1023193517090099

28. Maletzki F., Rosler H.-W., Staude E. J. // J. Membr. Sci., 1992, vol. 71, pp. 105-116. DOI: 10.1016/03767388(92)85010-G

29. Письменская Н. Д., Никоненко В. В., Белова Е. И., Лопаткова Г. Ю., Систа Ф., Пурсели Ж., Ларше К. // Электрохимия, 2007, т. 43, с. 325-345. DOI: 10.1134/S102319350703010X

30. Васильева В. И., Акберова Э. М., Жильцова А. В., Черных Е. И., Сирота Е. А., Агапов Б. Л. // Поверхность. Рентгеновские, синхротронные и нейтронные исследования, 2013, № 9, с. 27-34. DOI:10.1134/S102745101 3050194

31. Васильева В. И., Письменская Н. Д., Акберова Э. М., Небавская К. А. // Журн. физ. химии, 2014, т. 88, № 8, c. 1114-1120. DOI:10.1134/S0036024414080317

32. Сирота Е. А., Кранина Н. А., Васильева В. И., Малыхин М. Д., Селеменев В. Ф. // Вестник Воронежского государственного университета. Серия: Химия. Биология. Фармация, 2011, № 2, с. 53-59.

33. Васильева В. И., Жильцова А. В., Акберова Э. М., Фатаева А. И. // Конденсированные среды и межфазные гранииы, 2014, т. 16, № 3, с. 257-261. http://www.kcmf. vsu.ru/resources/t_16_3_2014_003.pdf

\title{
THE EFFECT OF THE DISPERSITY OF SULFOCATION-EXCHANGER OF HETEROGENEOUS MEMBRANES ON THE DEVELOPMENT OF DIFFUSION BOUNDARY LAYERS UNDER INTENSIVE CURRENT MODES
}

\author{
(C) 2018 E. M. Akberova, D. V. Kostylev, V. I. Vasil'eva \\ Voronezh State University, 1 Universitetskaya pl., 394018 Voronezh, Russia \\ e-mail: elmara_09@inbox.ru \\ Received 27.07.2018
}

\begin{abstract}
Recently, an idea regarding the surface optimization of ion-exchange membranes, aimed at increasing the overlimiting mass transfer by the development of heteroelectroconvection, has been extensively developed. Therefore, the purpose of the work is to conduct a laser-interferometric study of the effect which sulfocation-exchangers of experimental samples of heterogeneous Ralex CM Pes of varying dispersity have on the thickness of diffusion layers in a solution of the interphase boundary. Experimental samples of heterogeneous sulfocation-exchange membranes Ralex CM Pes ("MEGA" a.s., Czech Republic) were selected for the study. They were obtained by rolling a homogenized mixture of the milled ion-exchanger of various degrees of dispersity with polyethylene. The volume ratio of cation-exchanger to polyethylene was kept the same. The degree of dispersity of the sulfocationexchanger varied due to different milling times of 5 to 80 minutes. The experiments were performed in a seven-compartment electrodialysis cell with stable concentration-temperature stratification of the electrodialyzerin in a gravitational field. An interferometric setup was used to visualize the transport processes at the membrane-solution boundary.
\end{abstract}


A comparative analysis of the effect the electrical and geometric heterogeneity of the surface of the heterogeneous sulfocation-exchanger Ralex CM Pes membranes has on the conditions for the formation and development of diffusion layers under intense current modes was carried out. It was established that an increase in the milling time for the ion-exchanger particles leads to a decrease in the magnitude of the electrical inhomogeneity spacing of the surface and the fraction of macropores. Also, microrelief smoothing takes place. An increase in the time of the ion-exchanger milling from 5 to 80 min results in a 1.5 times decrease of the potential drop of the membrane. At this point the effective thickness of the diffusion layer begins to decrease. The study revealed the tendency of a decrease in the total and effective thickness of the diffusion layer with a decrease in the electrical inhomogeneity spacing of the membrane surface.

Keywords: heterogeneous sulfocation-exchange membrane, surface inhomogeneity, diffusion boundary layer, electroconvective instability, overlimiting current regimes.

DOI: https://doi.org/10.17308/kcmf.2018.20/572

\section{ACKNOWLEDGMENTS}

The work was supported by the grant of the President of the Russian Federation (grant number $M K-925.2018 .3)$.

The authors would like to thank MEGA a.s. (Czech Republic) and its owner Mr. L. Novak for providing experimental samples of the Ralex CM Pessulfocationexchange membranes.

The equipment of the Centre for Collective Use of Scientific Equipment of Voronezh State University was used to make microphotographs and AFM images of the membrane surface. URL: http://ckp.vsu.ru.

\section{REFERENCES}

1. Peers A. M. Discuss. Faraday Soc., 1956, vol. 21, pp. 124-125. DOI: 10.1039/DF9562100117

2. Newman J., Thomas-Alyea K. E. Electrochemical Systems. New York, John \& Sons, 2004, 672 p.

3. Urtenov M. A.-Kh., Kirillova E. V., Seidova N. M., Nikonenko V. V. J. Phys. Chem. B, 2007, vol. 111, no. 51, pp. 14208-14222. DOI: 10.1021/jp073103d

4. Kozmai A. E., Nikonenko V. V., Pismenskaya N. D., Pryakhina O. D., Sistat P., Pourcelly G. Russ. J. Electrochem., 2010, vol. 46, no. 12, pp. 1383-1389. DOI: 10.1134/ S1023193510120074

5. Larchet C., Nouri S., Auclair B., Dammak L., Nikonenko V., Adv. Colloid Interface Sci., 2008, vol. 139, pp. 45-61. DOI: 10.1016/j.cis.2008.01.007

6. Sistat P., Kozmai A., Pismenskaya N., Larchet C., Pourcelly G., Nikonenko V. Electrochim. Acta, 2008, vol. 53 , no. 22 , pp. $6380-6390$. DOI: $10.1016 /$ j.electacta.2008.04.041

7. Mareev S. A., Kozmay A. E., Pismenskaya N. D., Nikonenko V. V. Kondensirovannyye sredy $i$ mezhfaznye granitsy [Condensed Matter And Interphases], 2011, vol. 13, no. 2, pp. 172-177. Available at: http://www.kcmf.vsu.ru/ resources/t_13_2_2011_009.pdf (in Russ.)

8. Vasil'eva V. I., Shaposhnik V. A., Grigorchuk O. V. Russ. J. Electrochem., 2001, vol. 37, no. 11. pp. 1164-1171. DOI: $10.1023 / \mathrm{A}: 1012763531803$
9. Grigorchuk O. V., Vasil'eva V. I., Shaposhnik V. A. Desalination, 2005, vol. 184, pp. 431-438. DOI: 10.1016/ j.desal.2005.03.048

10. Shaposhnik V. A., Vasil'eva V. I., Grigorchuk O. V. Russ. J. Electrochem., 2006, vol. 42, no. 11, pp. 1202-1207. DOI: $10.1134 / \mathrm{S} 1023193506110061$

11. Vasil'eva V. I. Vestnik VGU, seriya: Khimiya. Biologiya. Farmatsiya [Proceedings of Voronezh State University. Series: Chemistry. Biology. Pharmacy], 2007, no. 1, pp. 359-379. (in Russ.)

12. Urtenov M. K., Uzdenova A. M., Kovalenko A. V., Nikonenko V. V., Pismenskaya N. D., Vasil'eva V. I., Sistat P., Pourcelly G. J. Membr. Sci., 2013, vol. 447, pp. 190202. DOI: 10.1016/j.memsci.2013.07.033

13. Nikonenko V. V., Vasil'eva V. I., Akberova E. M., Uzdenova A. M., Urtenov M. K., Kovalenko A. V., Pismenskaya N. P., Mareev S. A., Pourcelly G. Adv. Colloid Interface Sci., 2016, vol. 235, pp. 233-246. DOI: 10.1016/j. cis.2016.06.014

14. Pérez-Herranz V., Guiñón J. L., García-Antón J. // J. Applied Electrochem, 2000, vol. 30, iss. 7, pp. 809-816. DOI: 10.1023/A:100402242

15. Shahi V. K., Thampy S. K., Rangarajan R. Desalination, 2001, vol. 133, pp. 245-258. DOI: 10.1016/S00119164(01)00105-9

16. Vasil'eva V. I., Shaposhnik V. A., Grigorchuk O. V., Malykhin M. D. Russ. J. Electrochem, 2002, vol. 38, no. 8. pp. 846-852. DOI: 10.1023/A:1016809727673

17. Pawlowski S., Sistat Ph., Crespo J. G., Velizarov S. J. Membr. Sci., 2014, vol. 471, pp. 72-83. DOI: 10.1016/ j.memsci.2014.07.075

18. Shaposhnik V. A., Vasil'eva V. I., Grigorchuk O. V. Adv. Colloid Interface Sci., 2008, vol. 139, pp. 74-82. DOI: 10.1016/j.cis.2008.01.008

19. Tadimeti J. G. D., Kurian V., Chandra A., Chattopadhyay S. J. Membr. Sci., 2016, vol. 499, pp. 418-428. DOI: $10.1016 /$ j.memsci.2015.11.001

20. Gnusin N. P., Zabolotskiy V. I., Pismenskiy V. F., Litvinov S. P. Zhurn. prikl. khimii [Journal Applied Chemistry], 1979, vol. 52, pp. 1053-1058. (in Russ.)

21. Akberova E. M. Condensed Matter And Interphases, 2017, vol. 19, no. 3, pp. 314-320. Available at: 
http://www.kcmf.vsu.ru/resources/t_19_3_2017_001.pdf (in Russ.)

22. Berezina N. P., Kononenko N. A., Dvorkina G. A, Shel'deshov N. V. Fiziko-khimicheskiye svoystva ionoobmennykh materialov [Physico-Chemical Properties of IonExchange Materials]. Krasnodar: Izd-vo Kuban. gos. un-ta Publ., 1999, 82 p. (in Russ.)

23. Vasil'eva V. I., Shaposhnik V. A., Grigorchuk O. V., Petrunya I. P. Desalination, 2006, vol. 192, no. 1-3, pp. 408-414. DOI: 10.1016/j.desal.2005.06.055

24. Vasil'eva V., Zhiltsova A., Shaposhnik V., Zabolotsky V., Lebedev K., Malykhin M. "Ion Transport in Organic and Inorganic Membranes". Proceedings of Intern. Conf., 28 May-2 June, 2012, Krasnodar, 2012, pp. 233 235.

25. Vasil'eva V. I., Akberova E. M., Zabolotskii V. I. Russ. J. Electrochem., 2017, vol. 53, no. 4, pp. 398-410. DOI: $10.1134 / \mathrm{S} 1023193517040127$

26. Akberova E. M., Vasil'eva V. I., Malykhin M.D. Condensed Matter and Interphases, 2015, vol. 17, no. 3, pp. 273-280. Available at: http://www.kcmf.vsu.ru/resources/t_17_3_2015_002.pdf (in Russ.)

27. Nikonenko V. V., Mareev S. A., Pis'menskaya N. D., Uzdenova A. M., Kovalenko A. V., Urtenov M. Kh., Pourcelly G. Russ. J. Electrochem., 2017, vol. 53, no. 10, pp. 1122-1144. DOI: 10.1134/S1023193517090099
28. Maletzki F., Rosler H.-W., Staude E. J. J. Membr. Sci., 1992, vol. 71, pp. 105-116. DOI: 10.1016/03767388(92)85010-G

29. Pismenskaya N. D., Nikonenko V. V., Belova E. I., Lopatkova G. Yu., Sistat Ph., Pourcelly G., Larshe K. Russ. J. Electrochem., 2007, vol. 43, no. 3, pp. 307-327. DOI: 10.1134/S102319350703010X

30. Vasil'eva V. I., Akberova E. M., Zhiltsova A. V., Chernykh E. I., Sirota E. A., Agapov B. L. J. Surface Investigation. Xray, Synchrotron and Neutron Techniques, 2013, vol. 7, no. 5, pp. 833-840. DOI:10.1134/ S1027451013050194

31. Pismenskaya N. D., Akberova E. M., Nebavskaya K. A. Russ. J. Phys. Chem. A, 2014, vol. 88, no. 8, pp. 1293-1299. DOI:10.1134/S0036024414080317

32. Sirota E. A., Kranina N. A., Vasil'eva V. I., Malykhin M. D., Selemenev V. F. Vestnik VGU, seriya: Khimiya. Biologiya. Farmatsiya [Proceedings of Voronezh State University. Series: Chemistry. Biology. Pharmacy], 2011, no. 2, pp. 53-59. Available at: http://www.vestnik.vsu.ru/ program/view/view.asp? $\mathrm{sec}=$ chembio\&year $=2011 \&$ num $=$ 02\&f_name $=2011-02-08$ (in Russ.)

33. Vasil'eva V. I., Zhiltsova A. V., Akberova E. M., Fataeva A. I. Kondensirovannyye sredy i mezhfaznye granitsy [Condensed Matter And Interphases], 2014, vol. 16, no. 3, pp. 257-261. Available at: http://www.kcmf.vsu.ru/ resources/t_16_3_2014_003.pdf (in Russ.)
Акберова Эльмара Маликовна - к. х. н., ведущий инженер кафедры аналитической химии, Воронежский государственный университет; e-mail: elmara_09@inbox.ru

Костылев Денис Викторович - студент химического факультета, Воронежский государственный университет

Васильева Вера Ивановна - д. х. н., профессор кафедры аналитической химии, Воронежский государственный университет; e-mail: viv155@mail.ru
Elmara M. Akberova - Cand. Sci. (Chem.), Leading Engineer of the Analytical Chemistry Department, Voronezh State University; e-mail: elmara_09@inbox.ru

Denis V. Kostylev - the student of Chemical Faculty, Voronezh State University

Vera I. Vasil'eva - Dr. Sci. (Chem.), Professor of the Analytical Chemistry Department, Voronezh State University; e-mail: viv155@mail.ru 